\title{
Antipsychotic and antidepressant prescribing for 704297 children and young people with and without intellectual disabilities: record linkage study
}

Angela Henderson, Deborah Kinnear, Michael Fleming, Bethany Stanley, Nicola Greenlaw, Genevieve Young-Southward, Jill P. Pell and Sally-Ann Cooper

\section{Background}

Psychotropics are overprescribed for adults with intellectual disabilities; there are few studies in children and young people.

\section{Aims}

To investigate antipsychotic and antidepressant prescribing in children and young people with and without intellectual disabilities, and prescribing trends.

\section{Method}

Scotland's annual Pupil Census, which identifies pupils with and without intellectual disabilities, was record-linked to the Prescribing Information System. Antidepressant and antipsychotic data were extracted. Logistic regression was used to analyse prescribing between 2010 and 2013.

\section{Results}

Of the 704297 pupils, 16142 (2.29\%) had a record of intellectual disabilities. Antipsychotic and antidepressant use increased over time, and was higher in older pupils; antipsychotic use was higher in boys, and antidepressant use was higher in girls. Overall, antipsychotics were prescribed to 281 (1.74\%) pupils with intellectual disabilities and $802(0.12 \%)$ without (adjusted odds ratio 16.85, 95\% Cl 15.29-18.56). The higher use among those with intellectual disabilities fell each year (adjusted odds ratio 20.19 in 2010 v. 14.24 in 2013). Overall, 191 (1.18\%) pupils with intellectual disabilities and 4561 (0.66\%) without were prescribed antidepressants (adjusted odds ratio $2.28,95 \% \mathrm{Cl} 2.03$ 2.56). The difference decreased each year (adjusted odds ratio 3.10 in 2010 v. 2.02 in 2013).

\section{Conclusions}

Significantly more pupils with intellectual disabilities are prescribed antipsychotics and antidepressants than are other pupils. Prescribing overall increased over time, but less so for pupils with intellectual disabilities; either they are not receiving the same treatment advances as other pupils, or possible overprescribing in the past is changing. More longitudinal data are required.

\section{Keywords}

Intellectual disabilities; antipsychotics; antidepressants; children; young people.

\section{Copyright and usage}

(C) The Author(s), 2020. Published by Cambridge University Press on behalf of the Royal College of Psychiatrists. This is an Open Access article, distributed under the terms of the Creative Commons Attribution licence (http://creativecommons.org/ licenses/by/4.0/), which permits unrestricted re-use, distribution, and reproduction in any medium, provided the original work is properly cited.
Adults with intellectual disabilities are overprescribed psychotropic prescriptions, ${ }^{1,2}$, but surprisingly few studies have focussed on children and young people. Children and young people with intellectual disabilities are more likely to experience psychiatric disorders than those without intellectual disabilities. ${ }^{3-5}$ However, few children or young people experience psychosis, as the peak onset in males is at age 20-24 years and, for females, a less prominent peak occurs at $20-24$ years, followed by another at $>35$ years. ${ }^{6}$ However, antipsychotics are also prescribed 'off-label' in children and young people with intellectual disabilities with problem behaviours, such as aggression, disruptive behaviour or hyperactivity. ${ }^{7}$ Although there is some evidence of effectiveness, more so for risperidone, the quality of evidence is low, and side-effects are common, including for atypical antipsychotics. ${ }^{8-11}$ Indeed, it has been suggested that antipsychotic sideeffects occur more commonly in children with intellectual disabilities than in other children, and they have difficulty reporting them; ${ }^{3}$ it has also been suggested that prescribing antipsychotics to children with problem behaviours is maltreatment. ${ }^{12}$ The UK National Institute for Health and Care Excellence and other guidelines recommend that antipsychotic drugs should be reduced or discontinued in people with intellectual disabilities who are not experiencing psychosis, ${ }^{13,14}$ and that use is considered only when psychological or other interventions alone do not produce change within an agreed time, or risk is very severe. ${ }^{15}$ Similarly, systematic reviews of antidepressant prescribing in this population have also deemed studies to be low quality, with small sample sizes, ${ }^{16}$ and most antidepressants are contraindicated in children and youth, with suicidal ideation being a side-effect. Despite these cautions, there has been little study of the rates of prescription of antipsychotics and antidepressants in children with intellectual disabilities, or any changes over time. A review of general practice records in England reported that $2.4 \%$ with intellectual disabilities aged $\leq 18$ years were prescribed antipsychotics and $1.2 \%$ were prescribed antidepressants between 2009 and 2012, but the authors did not provide longitudinal data. ${ }^{1}$ A Taiwanese study reported an increase in out-patient clinic psychotropic prescribing between 1997 and 2007 of 16.67\% for children with intellectual disabilities, and $14.69 \%$ for youth with intellectual disabilities. ${ }^{17}$ For antidepressant prescribing, the increases were $46.05 \%$ for children with intellectual disabilities and $124.19 \%$ for youth with intellectual disabilities, but the sample was not population-based. ${ }^{17}$

\section{Aims}

This study aimed to investigate the use of antipsychotic and antidepressant prescribing to the whole population of children and young people with intellectual disabilities in Scotland, compared with those without intellectual disabilities, and to investigate trends in prescribing over a 4 -year period. 


\section{Method}

\section{Approvals}

This study was approval by the National Health Service (NHS) National Services Scotland Public Benefit and Privacy Panel (Application number 1617-0259). Individual participant consent was not required.

\section{Data sources and linkage}

In Scotland, a Pupil Census is conducted in September each year. It collects information on all children and young people attending local authority primary, secondary and special schools in Scotland, or children and young people with local authority-funded placements in alternative schools. This includes $95 \%$ of all children and young people in Scotland. We can identify no reason to suspect that children with intellectual disabilities would be more likely than other children to be home-educated or have parents paying school fees for their education at a public school. In 2010, 35.8\% of pupils with intellectual disabilities attended special schools, and $64.2 \%$ attended mainstream schools; in 2011, these figures were $29.1 \%$ and $70.9 \%$; in 2012, they were $27.7 \%$ and $72.4 \%$ and in 2013 , they were $29.0 \%$ and $71.0 \%$, respectively. The information collected in the Pupil Census includes whether the child/young person has a record of additional support needs, and the type of additional support needs including intellectual disabilities. The types of additional support needs recorded differentiate between intellectual disabilities and specific learning disabilities such as dyslexia and dyscalculia. Under the Education (Additional Support for Learning) (Scotland) Act 2004 (as amended), education authorities have a statutory requirement to identify and provide for the additional support needs of children and young people. The assessment includes informal observations by teachers, and more formal assessments from education, medical or psychological professionals (educational psychology, NHS community child health teams and clinical genetics services are available throughout Scotland, to provide these and other assessments). In the Pupil Census, teachers record the additional support needs identified from these assessments. It is held by the Scottish Exchange of Education Data. We used data from the four censuses conducted in 2010-2013, to identify children and young people with and without intellectual disabilities, aged up to 19 years. Records for each year were only included in the analysis if the child was aged 4 years or older in that particular year. We then used individual record linkage based on probabilistic record matching (on date of birth, gender and postcode) to the Community Health Index (CHI; Scotland's list of all unique patient identifiers). We excluded non-singleton births (identified from maternity records), as names were not used to link the pupil records to the $\mathrm{CHI}$, and therefore we could not decipher whether the correct child had linked. We excluded any records with duplicate pupil records or where the linkage was tied with another pupil. CHI was used to link the Pupil Census data at an individual level to prescribing data for each child; it did not provide any additional information for the study. Using the CHI, we then linked the Pupil Census data to Scotland's Prescribing Information System (PIS) to investigate prescriptions encashed over 2010-2013. PIS records all medicines that are prescribed and dispensed in the community in Scotland, or issued in hospitals and dispensed in the community, and prescriptions written in Scotland that were dispensed elsewhere in the UK, and includes coding of the prescriptions by British National Formulary codes. Only pupils with intellectual disabilities recorded in at least two different school years were included in the intellectual disabilities group, to reduce potential bias as a result of miscoding. Pupils who were included in at least two pupil censuses over the study period and had no record of intellectual disabilities were used as the comparison group. Antidepressant and antipsychotic prescribing data were extracted from the PIS for the two groups, for each of the 4 years (2010-2013). Prescription codes for each year were determined using the British National Formulary chapter 4, section code 402 for antipsychotics and 403 for antidepressants. ${ }^{18}$

\section{Statistical analyses}

Participant characteristics were summarised for pupils with and without intellectual disabilities, using counts and percentages for categorical data and mean and s.d. for age. Prescribing of antipsychotics and antidepressants at any time during 2010-2013 was summarised for pupils with and without intellectual disabilities by age group and gender, using counts and percentages. Prescribing was then compared between pupils with and without intellectual disabilities, using univariate logistic regression models to produce odds ratios and corresponding 95\% confidence intervals. The models were run univariately and then adjusted for age, gender and census year. These models were then extended again to include an interaction term between census year and population (intellectual disabilities or without intellectual disabilities), to assess whether there was a statistically significant difference in prescribing between census years for the different populations. If a statistically significant interaction was found, then subgroup analysis was performed by individual census years 2010 to 2013, and by population group (intellectual disabilities or without intellectual disabilities).

All analyses were performed with $\mathrm{R}$ version 3.5.0 for Windows (R Core team, Vienna, Austria; see https://www.R-project.org/).

\section{Results}

\section{Participant characteristics}

The final linked data-set contained records for 704297 pupils aged between 4 and 19 years, who had been included in at least two censuses. Of these, 16142 (2.29\%) had at least two records of additional support needs owing to intellectual disabilities, and 688155 (97.71\%) had no record of intellectual disabilities. The mean age of the pupils with intellectual disabilities was 9.53 years (s.d. 3.55), and the mean age of the pupils without intellectual disabilities was 9.22 years (s.d. 3.96). As expected, there were more male than female pupils with intellectual disabilities, ${ }^{19}$ and an almost even split in gender for the population without intellectual disabilities (Table 1).

\section{Antipsychotic prescribing}

Antipsychotics were prescribed at some point from 2010 to 2013 for $281(1.74 \%)$ of the pupils with intellectual disabilities and 802 $(0.12 \%)$ of the pupils without intellectual disabilities (Table 2). More male than female pupils were prescribed antipsychotics in

\begin{tabular}{|c|c|c|c|c|}
\hline & \multicolumn{2}{|c|}{ Intellectual disabilities } & \multicolumn{2}{|c|}{ No intellectual disabilities } \\
\hline & $\begin{array}{c}n \\
(n=16142)\end{array}$ & $\%$ & $\begin{array}{c}n \\
(n=688155)\end{array}$ & $\%$ \\
\hline \multicolumn{5}{|l|}{ Gender } \\
\hline Male & 10424 & $64.6 \%$ & 347539 & $50.5 \%$ \\
\hline Female & 5718 & $35.4 \%$ & 340616 & $49.5 \%$ \\
\hline \multicolumn{5}{|c|}{ Age in 2010 (years) } \\
\hline$<4$ & 554 & $3.4 \%$ & 56797 & $8.3 \%$ \\
\hline $4-11$ & 10292 & $63.8 \%$ & 398532 & $57.9 \%$ \\
\hline $12-16$ & 5201 & $32.2 \%$ & 231912 & $33.7 \%$ \\
\hline $17-19$ & 95 & $0.6 \%$ & 914 & $0.1 \%$ \\
\hline
\end{tabular}


Table 2 Antipsychotic and antidepressant prescribing in the period 2010-2013 in pupils with and without intellectual disabilities

\begin{tabular}{|c|c|c|c|c|}
\hline & \multicolumn{2}{|c|}{ Antipsychotics } & \multicolumn{2}{|c|}{ Antidepressants } \\
\hline & Intellectual disabilities, $n$ (\%) & Without intellectual disabilities, $n$ (\%) & Intellectual disabilities, $n$ (\%) & Without intellectual disabilities, $n$ (\%) \\
\hline All & 281/16 142 (1.74\%) & $802 / 688155(0.12 \%)$ & 191/16 142 (1.18\%) & $4561 / 688155(0.66 \%)$ \\
\hline \multicolumn{5}{|l|}{ Gender } \\
\hline Male & 204/10 424 (1.96\%) & 499/347 539 (0.14\%) & 111/10 424 (1.06\%) & 1424/347 539 (0.41\%) \\
\hline Female & $77 / 5718(1.35 \%)$ & $303 / 340616(0.09 \%)$ & 80/5718 (1.4\%) & $3137 / 340616$ (0.92\%) \\
\hline \multicolumn{5}{|c|}{ Age in 2010 (years) } \\
\hline 4-11 & 120/10 292 (1.17\%) & 282/398 $532(0.07 \%)$ & 55/10 292 (0.53\%) & $819 / 398532(0.21 \%)$ \\
\hline $12-16$ & 150/5201 (2.88\%) & $505 / 231912(0.22 \%)$ & 131/5201 (2.52\%) & $3693 / 231912$ (1.59\%) \\
\hline $17-19$ & $8 / 95$ (8.42\%) & $5 / 914(0.55 \%)$ & $<5 / 95(<5.22 \%)$ & $30 / 94$ (3.28\%) \\
\hline
\end{tabular}

both populations. The unadjusted odds ratio of the association of intellectual disabilities with antipsychotic prescribing was 18.21 (95\% CI 16.54-20.04)

Antipsychotics were prescribed to pupils with intellectual disabilities at rates of $0.85 \%$ in $2010,1.02 \%$ in $2011,0.90 \%$ in 2012 and $0.99 \%$ in 2013 ; compared with rates in pupils without intellectual disabilities of $0.04 \%$ in $2010,0.05 \%$ in $2011,0.05 \%$ in 2012 and $0.06 \%$ in 2013. Table 3 shows the logistic regression results, which indicated an odds ratio of 16.85 (95\% CI 15.29-18.56) of intellectual disabilities being associated with antipsychotic prescribing, when adjusted for census year, age and gender. Older age, male gender and later census year were also associated with an increase in antipsychotic prescribing (Table 3). The interaction term between population (intellectual disabilities or without intellectual disabilities) and census year was also significant $(P=0.002)$. Hence, subgroup analyses were conducted, and we found that the adjusted association of intellectual disabilities with being prescribed antipsychotics showed a gradient of falling over time (odds ratios of 20.19 (95\% CI 16.34-24.84) in 2010, 18.84 (95\% CI 15.57-22.70) in 2011, 15.55 (95\% CI 12.75-18.86) in 2012 and 14.24 (95\% CI 11.80-17.10) in 2013). In the subgroup analyses for the association of census year on antipsychotic prescribing in the population with intellectual disabilities, referenced to 2010, there was also a gradient showing increase over time (adjusted odds ratios of 1.18 (95\% CI $0.94-$ $1.49)$ for $2011,1.27$ (95\% CI 1.00-1.61) for 2012 and 1.80 (95\% CI 1.42-2.28) for 2013). For the population without intellectual disabilities, there was an increase over time; corresponding adjusted odds ratios were 1.29 (95\% CI 1.10-1.52) for 2011, 1.68 (95\% CI 1.43-1.98) for 2012 and 2.62 (95\% CI 2.24-3.08) for 2013. These subgroup analyses show that the increase in prescribing previously observed for year remains in both populations, most clearly for those without intellectual disabilities who have a greater increase with year.

\section{Antidepressant prescribing}

Antidepressants were prescribed at some point in 2010-2013 to 191 $(1.18 \%)$ of the pupils with intellectual disabilities, and $4561(0.66 \%)$ of the pupils without intellectual disabilities (Table 2). More female than male pupils were prescribed antidepressants in both populations. The unadjusted odds ratio of the association of intellectual disabilities with antidepressant prescribing was 2.05 (95\% CI 1.82-2.29).

Antidepressants were prescribed to pupils with intellectual disabilities at rates of $0.29 \%$ in $2010,0.47 \%$ in $2011,0.51 \%$ in 2012 and $0.60 \%$ in 2013; compared with rates in the pupils without intellectual disabilities of $0.10 \%$ in $2010,0.22 \%$ in $2011,0.25 \%$ in 2012 and $0.31 \%$ in 2013 . The logistic regression (Table 4), to determine the association between intellectual disabilities and antidepressant prescribing, found that pupils with intellectual disabilities were over twice as likely as other pupils to be prescribed antidepressants when adjusted for census year, age and gender. Older age, female gender and later census years were also associated with higher odds of antidepressant prescribing (Table 4). The interaction term between population (intellectual disabilities or without intellectual disabilities) and census year was significant $(P=0.007)$. Hence, subgroup analyses were conducted, and we found that the adjusted association of intellectual disabilities with being prescribed antidepressants showed a gradient of falling over time (odds ratios of 3.10 (95\% CI 2.27-4.12) in 2010, 2.48 (95\% CI 1.95-3.11) in 2011, 2.12 (95\% CI 1.69-2.64) in 2012 and 2.02 (95\% CI 1.64-2.47) in 2013). Similar to the antipsychotic results, this indicates that the increase in prescribing for the intellectual disabilities population overall remained across all years of the census, although the scale of the increased odds reduced by year. In the subgroup analyses exploring the association of census year on antidepressant prescribing in the intellectual disabilities population, referenced to census year 2010, there was also a gradient showing increase over time (adjusted odds ratios of 1.61 (95\% CI 1.12-2.33) for 2011, 2.27 (95\% CI 1.59-3.29) for 2012 and 3.82 (95\% CI 2.68-5.51) for 2013). For the population without intellectual disabilities, there was an increase over time; corresponding adjusted odds ratios were 2.14 (95\% CI 1.96-2.34) for 2011, 3.95 (95\% CI 3.61-4.31) for 2012 and 7.76 (95\% CI 7.10-8.49) for 2013. These subgroup analyses show that the increase in prescribing previously observed for year also remains in both populations, and is greater for those without intellectual disabilities.

Table 3 Logistic regression to determine the association of intellectual disabilities with antipsychotic prescribing

\begin{tabular}{|c|c|c|c|c|c|c|c|}
\hline & \multicolumn{3}{|c|}{ Unadjusted model } & \multicolumn{4}{|c|}{ Adjusted model $^{\mathrm{a}}$} \\
\hline & $\begin{array}{l}\text { Odds } \\
\text { ratio }\end{array}$ & $\begin{array}{l}\text { 95\% confidence } \\
\text { interval }\end{array}$ & $P$-value & $\begin{array}{l}\text { Odds } \\
\text { ratio }\end{array}$ & $\begin{array}{l}\text { 95\% confidence } \\
\text { interval }\end{array}$ & $P$-value & $\begin{array}{l}\text { Interaction } \\
P \text {-value }\end{array}$ \\
\hline Intellectual disabilities & 18.21 & $16.54-20.04$ & $<0.001$ & 16.85 & $15.29-18.56$ & $<0.001$ & \\
\hline Age (per 1-yr increase) & & & & 1.29 & $1.27-1.31$ & $<0.001$ & \\
\hline Female gender & & & & 0.53 & $0.48-0.58$ & $<0.001$ & \\
\hline Census year (per 1-yr increase) & & & & 1.32 & $1.27-1.38$ & $<0.001$ & \\
\hline $\begin{array}{l}\text { Interaction of census year and population (with or } \\
\text { without intellectual disabilities) }\end{array}$ & & & & & & & 0.002 \\
\hline
\end{tabular}




\begin{tabular}{|c|c|c|c|c|c|c|c|}
\hline & \multicolumn{3}{|c|}{ Unadjusted model } & \multicolumn{4}{|c|}{ Adjusted model $^{\mathrm{a}}$} \\
\hline & $\begin{array}{l}\text { Odds } \\
\text { ratio }\end{array}$ & $\begin{array}{c}\text { 95\% confidence } \\
\text { interval }\end{array}$ & $P$-value & $\begin{array}{l}\text { Odds } \\
\text { ratio }\end{array}$ & $\begin{array}{c}\text { 95\% confidence } \\
\text { interval }\end{array}$ & $P$-value & $\begin{array}{l}\text { Interaction } \\
P \text {-value }\end{array}$ \\
\hline Intellectual disabilities & 2.05 & $1.82-2.29$ & $<0.001$ & 2.28 & $2.03-2.56$ & $<0.001$ & \\
\hline Age (per 1-yr increase) & & & & 1.59 & $1.57-1.61$ & $<0.001$ & \\
\hline Female gender & & & & 1.99 & $1.89-2.10$ & $<0.001$ & \\
\hline Year (per 1-yr increase) & & & & 1.93 & $1.88-1.98$ & $<0.001$ & \\
\hline $\begin{array}{l}\text { Interaction of census year and population (with or } \\
\text { without intellectual disabilities) }\end{array}$ & & & & & & & 0.007 \\
\hline
\end{tabular}

\section{Discussion}

\section{Principal findings and interpretation}

As far as we are aware, this is the first study of antipsychotic and antidepressant prescribing, and trends in prescribing, in a country's whole population of children and young people with intellectual disabilities, in comparison with those without intellectual disabilities. Over the study period, $1.74 \%$ of pupils with intellectual disabilities received antipsychotics and $1.18 \%$ received antidepressants. Compared with their peers, their odds of receiving the drugs were 16 and 2, respectively, after adjusting for potential confounders. Psychotropic prescribing in children and young people must be undertaken cautiously after other options have been exhausted, but it does play a role in certain situations. The relative difference between them and their peers fell over time, but this was because of increased use of antipsychotics and antidepressants by their peers, not decreased use among children with intellectual disabilities. These apparent changes in antipsychotic, and particularly antidepressant use over a 4-year period highlight the need for further longitudinal studies, especially as there have been no other population-based longitudinal studies of psychotropic drug use conducted with children with intellectual disabilities.

\section{Comparison with previous literature}

Our finding of $1.74 \%$ of pupils with intellectual disabilities being prescribed antipsychotics in 2010-2013 compares with the higher rate reported in England of 2.4\% for the similar period of 20092012; however, our finding of $1.18 \%$ for antidepressants is similar to the $1.2 \%$ reported in England. ${ }^{1}$ We found that antipsychotic prescribing had increased, which was also reported in a study in Wales, but for a non-comparable population as it included children and youth with autism together with those with intellectual disabilities. ${ }^{20}$ The Taiwanese study reported considerable increase in prescribing of psychotropics, particularly antidepressants, but included only attenders at out-patient clinics, and has a different healthcare system to that in the UK, so is not comparable with our study. ${ }^{17}$ Additionally, in a study comparing 16 countries, Taiwan was shown to have the highest antipsychotic prescribing rates for general population children and young people of all the countries, so prescribing practices may differ from the UK. ${ }^{21}$ A small USA study is also not directly comparable with ours as they reported on children with comorbid intellectual disabilities and mental disorders; unlike our study, they found no change in antipsychotic use between 2007-2011.22 Psychotropic prescribing for children and young people has increased over time in the general population, particularly in Europe, the USA and Canada. ${ }^{23}$ In our study, we have shown that the extent of increase is less in pupils with intellectual disabilities than in pupils without intellectual disabilities.

Higher prescribing rates in youth rather than childhood mirrors the pattern of emerging mental disorders with age, and may be related to the greater challenges for families in managing aggressive problem behaviour in adolescents rather than smaller children. Higher prescribing rates of antipsychotics in males and antidepressants in females follow the same pattern as that seen in the general population, ${ }^{23}$ and in the English study of children and young people with intellectual disabilities. ${ }^{1}$

\section{Strengths and limitations}

Our study included the whole country, and the pupil census has approximately $95 \%$ coverage of school pupils. The data were longitudinal. Scotland is a high-income country, provides additional support in school to children with intellectual disabilities (which is advantageous to children with this diagnosis) and has comprehensive multidisciplinary community paediatric and genetic services, so few children with intellectual disabilities would not be identified. However, there may still be some recording errors in the census, hence we required the children to have a record of intellectual disabilities on at least two occasions to be included in the intellectual disabilities population. We did not have data on the severity of pupil's intellectual disabilities. We did not have data on the mental health of children and young people. Additionally, we were unable to access data on the $5 \%$ of children and young people not in school. Further, the pupil census is focussed on information about the pupils, not individual data about their parents or households, and so we have no contextual information that might have been relevant on any associations with, for example, parental mental health, parental income, parental occupation or parental educational attainments.

\section{Implications}

Children and young people with intellectual disabilities are significantly more likely to be prescribed antipsychotics and antidepressants than children and young people without intellectual disabilities. Psychotropic prescribing for children and young people is increasing, but at a lesser rate for those with intellectual disabilities compared with those without. We do not know if that suggests that children and young people with intellectual disabilities are not receiving the same treatment advances (i.e. better identification of mental ill-health and more prescribing, appropriately) over time as children and young people without intellectual disabilities, or if children and young people with intellectual disabilities were being overprescribed psychotropics in the past and this was addressed to an extent in this time period, or if overprescribing is increasing in children and young people without intellectual disabilities. It is important that prescribers in psychiatric services are vigilant to these possibilities, and prescribe when beneficial to the child, using the lowest therapeutic dose that is effective for each child, and withdrawing psychotropic drugs when there is no clear indication or benefit. Longitudinal observation is clearly important to quantify and understand these prescribing trends, and future research should 
investigate longitudinal prescribing in children and adolescents with intellectual disabilities in relation to mental health diagnoses, and adverse health outcomes.

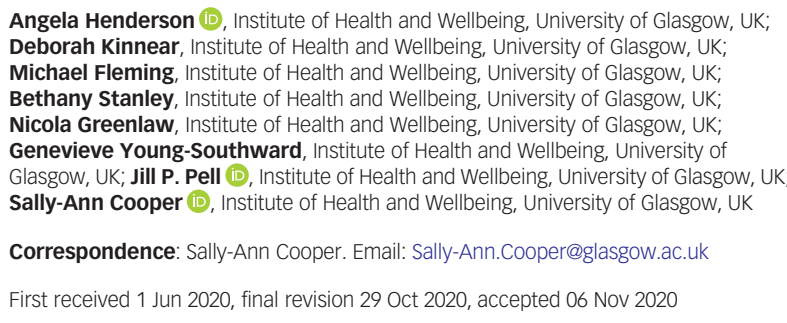

\section{Supplementary material}

To view supplementary material for this article, please visit https://doi.org/10.1192/bjp.2020.232.

\section{Data availability}

The researchers were authorised to receive data controlled and held by the Information Services Division, NHS National Services Scotland (NHS NSS), via access through the national safe haven. Data processing and sharing agreements were drafted with NHS NSS and ScotXed education. The Information Services Division Statistical Disclosure Control Protocol was followed. Therefore, data cannot be shared with other parties, but can be requested from the data controllers subject to the approval processes.

\section{Author contributions}

A.H., D.K. and S.-A.C. conceived the study. G.Y.-S. conducted the initial literature review. M.F. and J.J.P. developed the record linkage. B.S. and N.G. analysed the data. A.H. drafted the first version of the manuscript. All authors contributed to interpreting the data and writing the manuscript, and approved the final version of the manuscript.

\section{Funding}

The University of Glasgow was funded to undertake this study by the Medical Research Council (Mental Health Data Pathfinder Award, grant number MC_PC_17217).

\section{Declaration of interest}

None.

ICMJE forms are in the supplementary material, available online at https://doi.org/10.1192/ bjp.2020.232.

\section{References}

1 Glover G, Williams R. Prescribing of Psychotropic Drugs to People with Learning Disabilities and/or Autism by General Practitioners in England. Public Health England, 2015 (http://clok.uclan.ac.uk/17970/1/Psychotropic\%20medication\% 20and\%20people\%20with\%20learning\%20disabilities\%20or\%20autism.pdf).

2 Sheehan R, Hassiotis A, Walters K, Osborn D, Strydom A, Horsfall L. Mental illness, challenging behaviour, and psychotropic drug prescribing in people with intellectual disability: UK population based cohort study. BMJ 2015; 351: h4326.

3 Emerson E, Hatton C. Mental health of children and adolescents with intellectual disabilities in Britain. Br J Psychiatry 2007; 191: 493-9.

4 Einfeld SL, Ellis LA, Emerson E. Comorbidity of intellectual disability and mental disorder in children and adolescents: a systematic review. J Intellect Dev Disabil 2011; 36: 137-43.

5 Hughes-McCormack LA, Rydzewska E, Henderson A, Macintyre C, Rintoul J, Cooper SA. Prevalence of mental health conditions and relationship with general health in a whole-country population of people with intellectual disabilities compared with the general population. BJPSych Open 2017; 3(5): 243-8.

6 Jablensky A. Epidemiology and course of schizophrenia. In New Oxford Textbook of Psychiatry (3rd edn) (eds JR Geddes, NC Andreasen, GM Goodwin): 574-586. Oxford University Press, 2020

7 Handen BL, Gilchrist R. Practitioner review: psychopharmacology in children and adolescents with mental retardation. J Child Psychol Psychiatry 2006; 47(9): 871-82.

8 Unwin GL, Deb S. Efficacy of atypical antipsychotic medication in the management of behaviour problems in children with intellectual disabilities and borderline intelligence: a systematic review. Res Dev Disabil 2011; 32(6): 2121-33.

9 Matson JL, Neal D. Psychotropic medication use for challenging behaviors in persons with intellectual disabilities: an overview. Res Dev Disabil 2009; 30(3): 572-86.

10 Deb S, Farmah BK, Arshad E, Deb T, Roy M, Unwin GL. The effectiveness of aripiprazole in the management of problem behaviour in people with intellectual disabilities, developmental disabilities and/or autistic spectrum disorder-a systematic review. Res Dev Disabil 2014; 35(3): 711-25.

11 Solmi M, Fornaro M, Ostinelli EG, Zangani C, Croatto G, Monaco F, et al. Safety of 80 antidepressants, antipsychotics, anti-attention-deficit/hyperactivity medications and mood stabilizers in children and adolescents with psychiatric disorders: a large scale systematic meta-review of 78 adverse effects. World Psychiatry 2020; 19(2): 214-32.

12 Tsiouris JA. Pharmacotherapy for aggressive behaviours in persons with intellectual disabilities: treatment or mistreatment? J Intellect Disabil Res 2010; 54(1): 1-16.

13 National Institute for Health and Care Excellence (NICE). Mental Health Problems in People with Learning Disabilities: Prevention, Assessment and Management. NICE Guideline [NG54]. NICE, 2016 (https://www.nice.org.uk/ guidance/ng54).

14 Deb S, Kwok H, Bertelli M, Salvador-Carulla L, Bradley E, Torr J, et al. International guide to prescribing psychotropic medications for the management of problem behaviours in adults with intellectual disabilities. World Psychiatry 2009; 8: 181-6.

15 National Institute for Health and Care Excellence (NICE). Challenging Behaviour and Learning Disabilities: Prevention and Interventions for People with Learning Disabilities Whose Behaviour Challenges. NICE Guideline [NG11]. NICE, 2015 (https://www.nice.org.uk/guidance/ng11).

16 Sohanpal SK, Deb S, Thomas C, Soni R, Lenotre L, Unwin G. The effectiveness of antidepressant medication in the management of behaviour problems in adults with intellectual disabilities: a systematic review. J Intellect Disabil Res 2007; 51: 750-65.

17 Hsu SW, Chiang PH, Chang YC, Lin JD, Tung HJ, Chen CY. Trends in the use of psychotropic drugs in people with intellectual disability in Taiwan: a nationwide outpatient service study, 1997-2007. Res Dev Disabil 2014; 35(2): 36472.

18 Joint Formulary Committee. British National Formulary: 69. BMJ Group and Pharmaceutical Press, 2015

19 Cooper S-A. Epidemiology and course of intellectual disabilities. In New Oxford Textbook of Psychiatry (3rd edn) (eds J Geddes, N Andreasen, G Goodwin): 216-232. Oxford University Press, 2020.

20 Brophy S, Kennedy J, Fernandez-Gutierrez F, John A, Potter R, Linehan C, et al. Characteristics of children prescribed antipsychotics: analysis of routinely collected data. J Child Adol Psychopharmacol 2018; 28(3): 180-91.

21 Edelsohn GA, Schuster JM, Castelnovo K, Terhorst L, Parthasarathy M. Psychotropic prescribing for persons with intellectual disabilities and other psychiatric disorders. Psychiatr Serv 2014; 65(2): 201-7

22 Halfdanarson $\mathrm{O}$, Zoega $\mathrm{H}$, Aagaard L, Bernardo $\mathrm{M}$, Brandt L, Fuste AC, et al. International trends in antipsychotic use: a study in 16 countries, 2005-2014. Eur Neuropsychopharmacol 2017; 27(10): 1064-76.

23 Steinhausen HC. Recent international trends in psychotropic medication prescriptions for children and adolescents. Eur Child Adolesc Psychiatry 2015; 24 (6): 635-40. 